\title{
Transient Behavior of a System Composed of Conductive Thin Wire Structures Excited by Harmonic and Lightning Type Signals
}

\author{
Eduardo Faleiro', Gabriel Asensio², Daniel García3 ${ }^{3}$ Gregorio Denche3, Jorge Moreno ${ }^{3}$ \\ ${ }^{1}$ Departamento de Física Aplicada, ETSIDI, Universidad Politécnica de Madrid, Madrid, Spain \\ ${ }^{2}$ Departamento de Matemática Aplicada, ETSIDI, Universidad Politécnica de Madrid, Madrid, Spain \\ ${ }^{3}$ Departamento de Ingeniería Eléctrica, ETSIDI, Universidad Politécnica de Madrid, Madrid, Spain \\ Email: eduardo.faleiro@upm.es, gabriel.asensio@upm.es, daniel.gpuertas@upm.es, gdenche@ree.es, \\ jorge.moreno@upm.es
}

Received 17 July 2014; revised 13 August 2014; accepted 11 September 2014

Copyright (C) 2014 by authors and Scientific Research Publishing Inc.

This work is licensed under the Creative Commons Attribution International License (CC BY).

http://creativecommons.org/licenses/by/4.0/

c) (i) Open Access

\section{Abstract}

The transient response of a system of independent electrodes buried in a semi-infinite conducting medium is studied. Using a simple and versatile numerical scheme written by the authors and based on the Electric Field Integral Equation (EFIE), the effect caused by harmonic signals ranging on frequency from $\mathrm{Hz}$ to hundred of $\mathrm{MHz}$, and also by lightning type driving signal striking at a remote point far from the conductors, is extensively studied. The value of the scalar potential appearing on the electrodes as a function of the frequency of the applied signal is one of the variables investigated. Other features such as the input impedance at the injection point of the signal and the Ground Potential Rise (GPR) over the electrode system are also discussed.

\section{Keywords}

Transferred Potentials, Thin Wire Structures, Lightning Pulses, Electric Field Integral Equation, Method of Moments

\section{Introduction}

When a metallic conductor (the active electrode) is buried in a semi-infinite medium of non-zero conductivity, and is energized by either a voltage or a current signal variable in time, an electromagnetic field, which is pro- 
duced by the induced currents, is established within the electrode and into the surrounding medium which produces an elevation of the electrical potential in all the space and in particular on the ground surface or GPR, [1]-[4].

When metallic electrodes are located in the vicinity of the active electrode, even if they are not energized (passive electrodes), induced currents appear as a result of the interaction between them, all of which now form a system of interacting conductors and their potential will differ from the one they have when they are isolated or not part of the system [5] [6]. In this case we speak of transferred potentials by active to passive electrodes. This is particularly important since a metallic mass in low frequency regime can be considered equipotential, which means that a conductor can transmit the effect of belonging to a system of conductors to large distances compared with the characteristic size of the system. Suffice it to consider such a linear passive electrode of great length that is near a small casual energized electrode and to transfer a non-zero potential. This potential may appear in certain circumstances throughout the linear electrode at great distance from where the energization occurrs.

Everyday events that fit this simplified description happen when a fault current occurs in a grounding electrode of an electrical installation which is close to other grounding electrodes of other facilities that are initially isolated [7]. The release of the ground fault current and the subsequent appearance of an electromagnetic field lead to an electromagnetic coupling occurring between all adjacent metallic electrodes and the appearance of a transferred potential on each passive electrode [8].

In the present paper, based on the classic EFIE scheme proposed in [9], the authors have developed a simple and very versatile numerical code that is capable of handling complex systems with multiple electrodes and multiple feeding points for arbitrary time signals. Although there is commercial software that could run part of the calculations given in this paper, the authors have preferred to start from its own calculation scheme adapted to the problem under study.

The numerical code used is based upon the Moment Method as studied by Harrington [10], and can be applied to any electrode configuration composed of thin wires with any number of signal injection points working simultaneously [11]. Thus, it is possible to study the effect on the ground caused by the excitation of two buried electrodes of any shape and interconnected by an external conducting wire or, as in this work, the potential induced in the isolated passive electrodes of the system while only one of them is activated [12]-[14].

In this paper we consider a system of two electrodes, a simple square grid buried in the ground, the active electrode, and a square loop conductor placed on the ground surface above the first electrode and electrically isolated from the first one, the passive electrode. We will study the behavior of the potential transferred to the passive electrode as a function of the frequency of the signal applied.

The grounding impedance for the active electrode as a function of the excitation frequency is also studied, because it is considered as one of the most important parameters in the design of grounding systems. Finally we study the effect that causes an intense and short excitation lightning type signal applied directly to the active electrode and also at a remote point on the ground far from the electrode system [15] [16]. This is intended to simulate the effect of a lightning strike on the vicinity of a facility grounding system consisting of a buried wire mesh and a metallic fence on the surface surrounding the installation.

\section{Theoretical Foundations}

When a perfect conductor is buried in a semi-infinite medium of conductivity $\sigma$ and $\varepsilon$ and $\mu$ electromagnetic parameters and is subjected to an harmonic excitation of frequency $\omega$, the induced charges and currents act as sources of scalar $\phi$ and magnetic $\boldsymbol{A}$ potentials, so that the electric field induced by the harmonic signal and created by the structure is (Figure 1).

$$
\boldsymbol{E}(\boldsymbol{r})=-j \omega A(\boldsymbol{r})-\nabla \phi(\boldsymbol{r})
$$

Scalar and vector potentials for the case of filamentary conductors have an expression with significant simplifications. In fact, taking the arc length $s^{\prime}$ along the axis of the conductors as the independent variable.

$$
\phi(\boldsymbol{r}(s))=\int_{L^{\prime}} \frac{\rho_{s}\left(s^{\prime}\right)}{\varepsilon_{\text {eff }}} \cdot \frac{e^{-j k \cdot r(s)-r^{\prime}\left(s^{\prime}\right) \mid}}{4 \pi\left|\boldsymbol{r}(s)-\boldsymbol{r}^{\prime}\left(s^{\prime}\right)\right|} \mathrm{d} s^{\prime}
$$




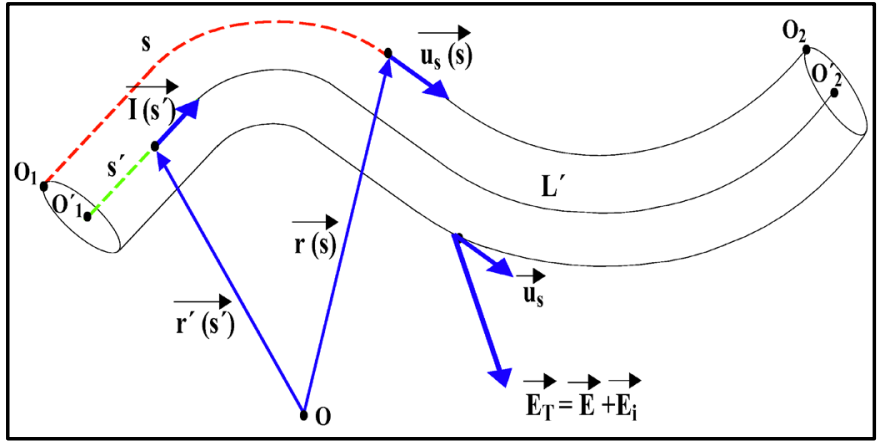

Figure 1. Curved thin wire conductor showing some of the variables used in the text.

$$
\boldsymbol{A}(\boldsymbol{r}(s))=\int_{L^{\prime}} \mu \cdot \boldsymbol{I}_{s}\left(s^{\prime}\right) \cdot \frac{e^{-j k \cdot \boldsymbol{r}(s)-\boldsymbol{r}^{\prime}\left(s^{\prime}\right) \mid}}{4 \pi\left|\boldsymbol{r}(s)-\boldsymbol{r}^{\prime}\left(s^{\prime}\right)\right|} \mathrm{d} s^{\prime}
$$

where everything is calculated along the axis of the wire, with the sole knowledge of the intensities $I_{s}$, along the conductor axis.

$$
\begin{gathered}
\varepsilon_{\mathrm{eff}}=\varepsilon+\frac{\sigma}{j \omega} \\
c=\frac{1}{\sqrt{\mu \varepsilon_{\mathrm{eff}}}}
\end{gathered}
$$

The calculation of currents is done by imposing the continuity of the tangential component of the total electric field, which is the sum of the exciting electric field and the induced electric field on the conductor surface. If the conductor is a PEC, the total electric field will be zero inside the conductor according to $\left(\boldsymbol{E}_{i}+\boldsymbol{E}\right) \cdot \boldsymbol{s}=0, \boldsymbol{s}$ being a unit vector tangent to the conductor surface.

From the expression on the continuity of the tangential component of the total field, it follows that

$$
\begin{aligned}
\boldsymbol{E}_{i} \cdot \boldsymbol{s} & =-\boldsymbol{E} \cdot \boldsymbol{s}=E_{i}(s) \\
& =j \omega \mu \cdot \boldsymbol{s} \cdot \int_{L^{\prime}} I\left(s^{\prime}\right) \boldsymbol{s}^{\prime} G\left(s, s^{\prime}\right) \mathrm{d} s^{\prime}-\boldsymbol{s} \cdot \nabla \int_{L^{\prime}} \frac{1}{j \omega \varepsilon_{\text {eff }}}\left[\frac{\partial}{\partial s^{\prime}} I\left(s^{\prime}\right)\right] G\left(s, s^{\prime}\right) \mathrm{d} s^{\prime} .
\end{aligned}
$$

where the transient kernel has been introduced

$$
G\left(s, s^{\prime}\right)=\frac{e^{-j k \cdot\left|r(s)-r^{\prime}\left(s^{\prime}\right)\right|}}{4 \pi\left|\boldsymbol{r}(s)-\boldsymbol{r}^{\prime}\left(s^{\prime}\right)\right|}
$$

and the approximation has been used,

$$
\left.\nabla^{\prime} \cdot \boldsymbol{J}\left(\boldsymbol{r}^{\prime}\right)\right|_{e j e}=\frac{\partial}{\partial s^{\prime}} I\left(s^{\prime}\right)=-j \omega \rho\left(\boldsymbol{r}^{\prime}\right)
$$

In order to work exclusively with the longitudinal currents $I(s)$, an integration by parts is done

$$
E_{i}(s) \cdot j \omega \varepsilon_{\text {eff }}=-k^{2} \int_{L^{\prime}} I\left(s^{\prime}\right) s \cdot s^{\prime} G\left(s, s^{\prime}\right) \mathrm{d} s^{\prime}-\boldsymbol{s} \cdot \nabla\left[\int_{L^{\prime}}\left[\frac{\partial}{\partial s^{\prime}}\left(I\left(s^{\prime}\right) G\left(s, s^{\prime}\right)\right)-I\left(s^{\prime}\right) \frac{\partial G\left(s, s^{\prime}\right)}{\partial s^{\prime}}\right] \mathrm{d} s^{\prime}\right]
$$

The second term of the RHS can be calculated by introducing the expression

$$
\operatorname{Cont}(s)=\left(I\left(s^{\prime}\right) G\left(s, s^{\prime}\right)\right)_{s^{\prime}=i}^{s^{\prime}=d}=I(d) G(s, d)-I(i) G(s, i)
$$

which is a function of the variable $s$, and must be evaluated by $s^{\prime}$ for the left end and for the right end of the wire. We shall call this expression, boundary conditions of the wire. These boundary conditions detail the elec- 
trical behavior of the free ends of the wire. If there is no input or output current by the ends of the wire, or the wire itself is a loop, then $\operatorname{Cont}(s)=0$ and there is no contribution to the whole expression.

Considering these details,

$$
E_{i}(s) \cdot j \omega \varepsilon_{\text {eff }}=-k^{2} \int_{L^{\prime}} I\left(s^{\prime}\right) s \cdot s^{\prime} G\left(s, s^{\prime}\right) \mathrm{d} s^{\prime}+\boldsymbol{s} \cdot \nabla \int_{L^{\prime}} I\left(s^{\prime}\right) \frac{\partial}{\partial s^{\prime}} G\left(s, s^{\prime}\right) \mathrm{d} s^{\prime}-\frac{\partial}{\partial s} \operatorname{Cont}(s)
$$

but it is easy to check that $s \cdot \nabla \equiv \frac{\partial}{\partial s}$, so that the above expression can be reduced to

$$
E_{i}(s) \cdot j \omega \varepsilon_{\mathrm{eff}}=\int_{L^{\prime}} I\left(s^{\prime}\right)\left[\frac{\partial^{2}}{\partial s \partial s^{\prime}} G\left(s, s^{\prime}\right)-k^{2} G\left(s, s^{\prime}\right) s \cdot s^{\prime}\right] \mathrm{d} s^{\prime}-\frac{\partial}{\partial s} \operatorname{Cont}(s)
$$

which, in even more compacted, results

$$
E_{i}(s) \cdot j \omega \varepsilon_{\text {eff }}=\int_{L^{\prime}} I\left(s^{\prime}\right) K\left(s, s^{\prime}\right) \mathrm{d} s^{\prime}-\frac{\partial}{\partial s} \operatorname{Cont}(s)
$$

The Expression (13) is an integral equation for the variable $I\left(s^{\prime}\right)$, which represents the longitudinal currents distribution at the position $s^{\prime}$ on the electrode axis. This expression can be used broadly not only for any type of electrode to which the thin wire approach can be applied, but it also may be applied to systems consisting of different not connected electrodes for which this thin wire approach is valid. The conductor system described here, can be excited with separate signals for each conductor and the longitudinal currents system calculated for each one. In this case, the integral in (13) spans on all the conductors, and the boundary conditions term contains the currents across all the free ends of the whole system.

\section{Numerical Solution and Application to Complex Systems}

With the aim to find the distribution of longitudinal currents $I\left(s^{\prime}\right)$ along the conductor system to which the thin-wire approximation is applied, the Method of Moments is used.

The Method of Moments is a numerical procedure that allows obtaining a solution to Equation (13) by reducing the integral equation to a matrix equation as defined by Harrington [10]. To do this, the overall curved wire of length $L$, is divided into $N$ small sized line segments $\Delta s^{\prime}=L / N$. A selected set of known functions is chosen to build up a linear approximation to the unknown function $I\left(s^{\prime}\right)$,

$$
I\left(s^{\prime}\right)=\sum_{n=1}^{N} I_{n} \cdot u_{n}\left(s^{\prime}\right)
$$

as an example, the unit step function can be chosen for $u_{n}\left(s^{\prime}\right)$,

$$
\begin{array}{ll}
u_{n}\left(s^{\prime}\right)=1 & \text { if }(n-1) \Delta s^{\prime}<s^{\prime}<n \Delta s^{\prime}, \\
u_{n}\left(s^{\prime}\right)=0 & \text { otherwise. }
\end{array}
$$

Note that this is a staircase-like approximation to the function $I\left(s^{\prime}\right)$, for which $I\left(s^{\prime}=0\right)=I_{0}$, while $I\left(s^{\prime}=L^{\prime}\right)=I_{N+1}, I_{0}$ and $I_{N+1}$ being the currents flowing to the left and right of the wire, respectively, according with the step functions in (14) and (15).

The choice of these functions $u_{n}$ is made for simplicity, but they are neither the only possible nor the best numerical results to be provided. In this paper the Function (14) were chosen because of their simplicity at the risk of losing some accuracy in the final results [10] [11].

Introducing the (14) into (13) and taking into account the definition of the basis functions it follows that,

$$
E_{i}(s) \cdot j \omega \varepsilon_{\text {eff }}=\sum_{n=1}^{N} I_{n} \cdot \int_{(n-1) \Delta s^{\prime}}^{n \Delta s^{\prime}} K\left(s, s^{\prime}\right) \mathrm{d} s^{\prime}-\frac{\partial}{\partial s} \operatorname{Cont}(s)
$$

expression valid at any point $s$ on the electrode. Finally, a set of $N$ weighting functions are being used, such as the Dirac delta function (point matching method) according to [11],

$$
w_{m}(s)=\delta\left(s-s_{m}\right)
$$


where, the points $s_{m}$ are on the electrode surface and are chosen arbitrarily. It is usual that they are aligned perpendicular to the midpoints of the segments $\Delta s^{\prime}$ on the axis wire,

$$
j \omega \varepsilon_{\mathrm{eff}} \int_{L} E_{i}(s) \delta\left(s-s_{m}\right) \mathrm{d} s=\sum_{n=1}^{N} I_{n} \cdot \int_{L} \delta\left(s-s_{m}\right)\left[\int_{(n-1) \Delta s^{\prime}}^{n s^{\prime}} K\left(s, s^{\prime}\right) \mathrm{d} s^{\prime}\right] \mathrm{d} s-\int_{L} \delta\left(s-s_{m}\right) \frac{\partial}{\partial s} \operatorname{Cont}(s) \mathrm{d} s
$$

This gives us a system of $N$ equations with $N$ unknowns, the current $I_{n}$,

$$
E_{i}\left(s_{m}\right) \cdot j \omega \varepsilon_{\text {eff }}=\sum_{n=1}^{N} I_{n} \cdot \int_{(n-1) \Delta s^{\prime}}^{n \Delta s^{\prime}} K\left(s_{m}, s^{\prime}\right) \mathrm{d} s^{\prime}-\left[\frac{\partial}{\partial s} \operatorname{Cont}(s)\right]_{s=s_{m}}
$$

The use of Dirac delta functions is not unique. If functions $u_{n}(s)$ are also used as weighting functions, it will be obtained on the electrode surface for each $n=1, \cdots, N$,

$$
j \omega \varepsilon_{\text {eff }} \int_{L} E_{i}(s) u_{n}(s) \mathrm{d} s=\sum_{n=1}^{N} I_{n} \cdot \int_{L} u_{n}(s)\left[\int_{(n-1) \Delta s^{\prime}}^{n \Delta s^{\prime}} K\left(s, s^{\prime}\right) \mathrm{d} s^{\prime}\right] \mathrm{d} s-\int_{L} u_{n}(s) \frac{\partial}{\partial s} \operatorname{Cont}(s) \mathrm{d} s
$$

This procedure is known as Galerkin method and its main advantage is to ensure the electrode to be equipotential unlike the point matching method, in which only it is ensured that the electrode is equipotential at $s_{m}$ points.

For the Galerkin method, the above expression is,

$$
j \omega \varepsilon_{\text {eff }} \int_{(n-1) \Delta s}^{n \Delta s} E_{i}(s) \mathrm{d} s=\sum_{n=1}^{N} I_{n} \cdot \int_{(n-1) \Delta s}^{n \Delta s} \int_{(n-1) \Delta s^{\prime}}^{n \Delta s^{\prime}} K\left(s, s^{\prime}\right) \mathrm{d} s^{\prime} \mathrm{d} s-\int_{(n-1) \Delta s}^{n \Delta s} \frac{\partial}{\partial s} \operatorname{Cont}(s) \mathrm{d} s
$$

By application of the point matching method, considering the kernel $K$ given in (13) and applying the finite increments approach to derivatives, we obtain the expression,

$$
j \omega \varepsilon_{\mathrm{eff}} E_{i}\left(s_{m}\right) \cdot \Delta s=\sum_{n=1}^{N} I_{n} \cdot\left[\chi(m, n)-k^{2} \cdot \Delta s \Delta s^{\prime} \cdot \boldsymbol{s}_{m} \cdot \boldsymbol{s}_{n}^{\prime} \cdot \Psi(m, n)\right]-\left[\frac{\partial}{\partial s} \operatorname{Cont}(s)\right]_{s=s_{m}}
$$

where we have introduced the notation

$$
\begin{gathered}
\chi(m, n)=\left(G_{++}(m, n)-G_{+-}(m, n)-G_{-+}(m, n)+G_{--}(m, n)\right) \\
\Psi(m, n)=\frac{1}{\Delta s^{\prime}} \int_{(n-1) \Delta s^{\prime}}^{n \Delta s^{\prime}} G\left(s_{m}, s^{\prime}\right) \mathrm{d} s^{\prime}
\end{gathered}
$$

in which we have established the following criteria: the segments are oriented from left to right assigning the left end to $(n-1) \Delta s^{\prime}$ and the symbol " -", and the right end to $n \Delta s^{\prime}$ and the symbol " + ". Thus, for example $G_{-+}(m, n)$ means that we have to evaluate the kernel $G\left(s, s^{\prime}\right)$ for s at the left end of the field segment and $s^{\prime}$ at the right end of the source segment.

If we define the mutual impedance matrix as

$$
Z(m, n)=\frac{E_{i}\left(s_{m}\right) \cdot \Delta s}{I_{n}}
$$

and remembering that $k^{2}=\omega^{2} \mu \varepsilon_{\text {eff }}$, it follows that

$$
Z(m, n)=\frac{1}{j \omega \varepsilon_{\mathrm{eff}}} \chi(m, n)+j \omega \mu \cdot \Delta \boldsymbol{s}_{m} \cdot \Delta \boldsymbol{s}_{n}^{\prime} \cdot \Psi(m, n)
$$

Since $E_{i}\left(s_{m}\right) \cdot \Delta s$ represents the potential drop due to the electric field incident on the surface of the segment whose midpoint is $s_{m}$, then

$$
\Delta V_{i}(m)=\sum_{n=1}^{N} I_{n} \cdot Z(m, n)-\frac{1}{j \omega \varepsilon_{\text {eff }}}\left[\frac{\partial}{\partial s} \operatorname{Cont}(s)\right]_{s=s_{m}}, \quad m=1, \cdots, N .
$$

which is a linear system of $N$ equations with $N$ unknowns, homogeneous for a PEC, and shall therefore incorporate relations between the currents that define the boundary conditions (potential free ends of the wires 
where the current vanishes) and the feeding points if any. These are given by a known current value applied to a junction point (node) between parts of the structure. It is easy to see that,

$$
\left[\frac{\partial}{\partial s} \operatorname{Cont}(s)\right]_{s=s_{m}}=I_{N+1}\left(G_{++}(m, N)-G_{-+}(m, N)\right)-I_{0}\left(G_{+-}(m, 1)-G_{--}(m, 1)\right)
$$

whose contribution to (27) is

$$
\Delta V_{i}(m)=\sum_{n=1}^{N} I_{n} \cdot Z(m, n)-I_{N+1} \frac{1}{j \omega \varepsilon_{\text {eff }}}\left(G_{++}(m, N)-G_{-+}(m, N)\right)+I_{0} \frac{1}{j \omega \varepsilon_{\text {eff }}}\left(G_{+-}(m, 1)-G_{--}(m, 1)\right)
$$

which is valid for all $m=1, \cdots, N$, field points. With respect to the feeding points, it can be easily proved that for a rod divided into $N$ portions with open ends and a feeding point $I_{p i}$ on the right side of the segment $n_{i}$, the Expression (29) is

$$
\begin{aligned}
\Delta V_{i}(m)= & \sum_{n=1}^{N} I_{n} \cdot Z(m, n)-I_{N+1} \frac{1}{j \omega \varepsilon_{\text {eff }}}\left(G_{++}(m, N)-G_{-+}(m, N)\right)+I_{0} \frac{1}{j \omega \varepsilon_{\text {eff }}}\left(G_{+-}(m, 1)-G_{--}(m, 1)\right) \\
& +I_{p i} \frac{1}{j \omega \varepsilon_{\text {eff }}}\left(G_{++}\left(m, n_{i}\right)-G_{-+}\left(m, n_{i}\right)\right) .
\end{aligned}
$$

There only remains to be added the boundary condition associated with semi-infinite nature of the surroundings. For a perfectly horizontal surface that separates the conductive soil from the air, just consider the existence of a virtual electrode located symmetrically with respect to the horizontal ground surface and in an identical electrical state as the actual electrode. Currents must flow in such a way that they should be a mirror reflection of the actual ones with respect to the horizontal ground.

Under these conditions, the potential can be calculated correctly in any point at the ground. This does not apply to air, where the virtual electrode is located.

We introduce the mutual impedances by,

$$
Z(m, n)=\frac{1}{j \omega \varepsilon_{\mathrm{eff}}} \chi(m, n)+j \omega \mu \cdot \Delta \boldsymbol{s}_{m} \cdot \Delta \boldsymbol{s}_{n}^{\prime} \cdot \Psi(m, n)++\frac{F}{j \omega \varepsilon_{\mathrm{eff}}} \chi\left(m, n_{I}\right)+j \omega \mu F \cdot \Delta \boldsymbol{s}_{m} \cdot \Delta \boldsymbol{s}_{n_{I}}^{\prime} \cdot \Psi\left(m, n_{I}\right)
$$

where $\mathrm{F}$ is a correction factor that takes into account the electrical parameters of the two media. For a rank of $|k \cdot R| \ll 1$, the value of $F$ is

$$
F=\frac{\varepsilon_{\text {eff }}(\text { soil })-\varepsilon_{\text {eff }}(\text { air })}{\varepsilon_{\text {eff }}(\text { soil })+\varepsilon_{\text {eff }}(\text { air })}=\frac{\varepsilon_{\text {eff }}-\varepsilon_{0}}{\varepsilon_{\text {eff }}+\varepsilon_{0}}
$$

since the electrical conductivity of air is nearly zero, $\sigma($ air $)=0$.

In the above formula, $n_{I}$ refers to the source segment that is the image of the segment $n$ belonging to the actual electrode, i.e.

$$
\chi\left(m, n_{I}\right)=G_{++}\left(m, n_{I}\right)-G_{+-}\left(m, n_{I}\right)-G_{-+}\left(m, n_{I}\right)+G_{--}\left(m, n_{I}\right)
$$

In order to build the matrix of the complete system, including free ends and injection points, we extend the initial matrix size $N \times N$ to include " $e l$ " free ends and " $p i$ " direct injection points in the structure, so that the entire array has a size $(N+e l+p i) \times(N+e l+p i)$. Obviously, the number of unknowns will have increased up to $N+e l+p i$. The data that need to be known in order to reach the solution are the "el" currents at the free ends and the " $p i$ " currents in the injection points.

$$
\begin{aligned}
\Delta V_{i}(m)= & \sum_{n=1}^{N} I_{n} \cdot Z(m, n)+\sum_{l=1}^{e l} \frac{1}{j \omega \varepsilon_{\mathrm{eff}}}\left[I_{0_{l}}\left(G_{+-}\left(m, 1_{l}\right)-G_{--}\left(m, 1_{l}\right)\right)-I_{N_{l}+1}\left(G_{++}\left(m, N_{l}\right)-G_{-+}\left(m, N_{l}\right)\right)\right] \\
& +\sum_{e=1}^{p i} \frac{1}{j \omega \varepsilon_{\mathrm{eff}}} I_{i_{e}}\left(G_{++}\left(m, n_{i}\right)-G_{-+}\left(m, n_{i}\right)\right)+\sum_{n=1}^{N} I_{n} \cdot F \cdot Z\left(m, n_{I}\right) \\
& +F \cdot \sum_{l=1}^{e l} \frac{1}{j \omega \varepsilon_{\mathrm{eff}}}\left[I_{0_{l}}\left(G_{+-}\left(m, 1_{I l}\right)-G_{--}\left(m, 1_{I l}\right)\right)-I_{N_{l}+1}\left(G_{++}\left(m, N_{I l}\right)-G_{-+}\left(m, N_{I l}\right)\right)\right] \\
& +F \cdot \sum_{e=1}^{p i} \frac{1}{j \omega \varepsilon_{\mathrm{eff}}} I_{i_{e}}\left(G_{++}\left(m, n_{l i}\right)-G_{-+}\left(m, n_{l i}\right)\right) .
\end{aligned}
$$


Finally, we propose a matrix impedances that is organized as

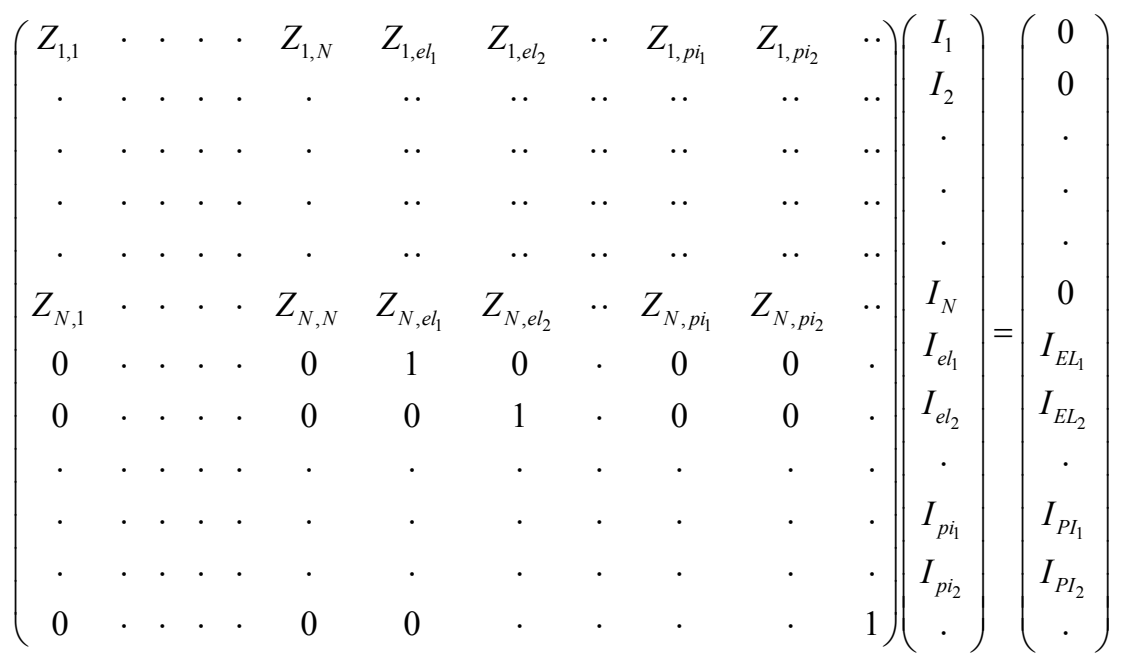

where the column vector of the RHS are known data corresponding to incoming-outgoing currents at the free ends $I_{E L}$, and the currents at the direct injection points $I_{P I}$. We should not forget that the zeros of the RHS vector are due to the electrode being a PEC. Otherwise, it is necessary to consider the internal impedance of the electrode. The matrix elements are.

$$
\begin{aligned}
Z_{m, n} & =Z(m, n)+F \cdot Z\left(m, n_{I}\right), \\
Z_{m, e l} & =\frac{1}{j \omega \varepsilon_{\text {eff }}}\left(G_{+-}\left(m, 1_{e l}\right)-G_{--}\left(m, 1_{e l}\right)\right)+F \cdot \frac{1}{j \omega \varepsilon_{\text {eff }}}\left(G_{+-}\left(m, 1_{I e l}\right)-G_{--}\left(m, 1_{I e l}\right)\right) \\
& =-\frac{1}{j \omega \varepsilon_{\text {eff }}}\left(G_{++}\left(m, N_{e l}\right)-G_{-+}\left(m, N_{e l}\right)\right)-F \cdot \frac{1}{j \omega \varepsilon_{\text {eff }}}\left(G_{++}\left(m, N_{I e l}\right)-G_{-+}\left(m, N_{I e l}\right)\right), \\
Z_{m, p i} & =\frac{1}{j \omega \varepsilon_{\text {eff }}}\left(G_{++}\left(m, n_{p i}\right)-G_{-+}\left(m, n_{p i}\right)\right)+F \cdot \frac{1}{j \omega \varepsilon_{\text {eff }}}\left(G_{++}\left(m, n_{I p i}\right)-G_{-+}\left(m, n_{I p i}\right)\right) .
\end{aligned}
$$

If the structure has no free ends being a loop, the impedances $Z_{m, e l}$ are no relevant quantities, because in the RHS vector of currents all the free ends currents will be zero. In a closed structure without free ends, the excitation of the electrode can be made from direct feeding points.

Once determined the distributions of charges and currents in the buried electrode, the relevant magnitudes and the electrical parameters can be calculated at any point in the ground. One of the most common ones is the absolute potential with respect to far earth at any point on the surface of the soil,

$$
\phi(\boldsymbol{r}(s))=\int_{L^{\prime}}-\frac{1}{j \omega \varepsilon_{\text {eff }}}\left[\frac{\partial}{\partial s^{\prime}} I\left(s^{\prime}\right)\right] \cdot \frac{e^{-j k \cdot r(s)-\boldsymbol{r}^{\prime}\left(s^{\prime}\right) \mid}}{4 \pi\left|\boldsymbol{r}(s)-\boldsymbol{r}^{\prime}\left(s^{\prime}\right)\right|} \mathrm{d} s^{\prime}
$$

Using the entire set of operations made before, as the integration by parts, the method of moments for the numerical approximation, the introduction of the feeding points and discontinuity of the ground surface, a final expression is obtained,

$$
\begin{aligned}
\phi(r(s))= & \frac{1}{j \omega \varepsilon_{\text {eff }}} \sum_{n=1}^{N} I_{n}\left[G\left(R_{n+}\right)-G\left(R_{n-}\right)\right]-\frac{1}{j \omega \varepsilon_{\text {eff }}}\left(I_{N+1} G\left(R_{N+}\right)-I_{0} G\left(R_{1-}\right)\right)+\frac{1}{j \omega \varepsilon_{\text {eff }}} I_{p i} G\left(R_{p i+}\right) \\
& +\frac{F}{j \omega \varepsilon_{\text {eff }}} \sum_{n=1}^{N} I_{n}\left[G\left(R_{n_{I}+}\right)-G\left(R_{n^{-}}\right)\right]-\frac{F}{j \omega \varepsilon_{\text {eff }}}\left(I_{N+1} G\left(R_{N_{I^{+}}}\right)-I_{0} G\left(R_{1_{I}-}\right)\right)+\frac{F}{j \omega \varepsilon_{\text {eff }}} I_{p i} G\left(R_{p i_{I}+}\right) .
\end{aligned}
$$

where the following abbreviation it is introduced

$$
G\left(R_{n \pm}\right)=\frac{e^{-j k R_{n \pm}}}{4 \pi R_{n \pm}}
$$


$R_{n \pm}$ being the distance between the right (left) end of the wire segment $n$ and the field point.

\section{Induced Potentials in the Electrode System}

We will apply the above theoretical framework to the study of a system consisting of two conductors as shown in Figure 2. The lower regular mesh conductor, which will be called active conductor, which can be energized through the injection points IP1 and IP2 marked in Figure 2. The upper conductor, denominated as the passive conductor, is placed symmetrically on the active conductor and at the ground surface. Both conductors represent a simplified model of grounding protection facilities. In this model, the passive conductor simulates a metal fencing surrounding the facility.

First, the active conductor is energized by a defined frequency harmonic signal. With the help of the above theoretical expressions, the potential acquired by both electrodes will be calculated as a function of the frequency of the signal injected on the active electrode.

We are mainly interested in calculating the potential of the passive electrode, which simulates a metal fence surrounding an area electrically protected by the buried grid.

Potentials along point $\mathrm{p} 2$ to $\mathrm{p} 4$ of the buried grid and along the contour $\mathrm{OABC}$ on the passive electrode will be calculated at several frequencies.

The representative value of the potential will be its peak value, associated with the harmonic behavior of the electrode response.

The grounding impedance, as a function of the frequency, will be also calculated at the two feeding points IP1 and IP2.

Next, a fast and peaked pulse of electrical current is injected into the buried grid through the feeding point IP2 and the potential as a function of time is estimated on the points $\mathrm{p} 3$ and $\mathrm{p} 4$ of the active electrode. The same is done at the point $\mathrm{p} 5$ on the passive electrode. Finally, another fast and peaked pulse is injected into the ground surface far from the system of conductors at the external injection point EIP of Figure 2. The potential at points $\mathrm{p} 1$ to $\mathrm{p} 5$ is evaluated as a function of time. For both pulses, the potential appearing at the ground surface along the diagonal $\mathrm{OB}$ on the passive electrode is also evaluated.

The calculations were carried out for a $4 \mathrm{~m}$ length $\mathrm{L}$ of the upper conductor and $1 \mathrm{~m}$ length 1 for the wires on the bottom grid. The burial depth $h$ is $0.5 \mathrm{~m}$ and the distance between the injection point EIP and the surface conductors $\mathrm{D}$ as seen in Figure 2, ranks from $2 \mathrm{~m}$ to $8 \mathrm{~m}$.

In all calculations, the electrodes are initially divided into $N=20$ segments per meter of conductor. It was considered that the ground has a conductivity of $100 \Omega \cdot \mathrm{m}$ and a value of 10 in the relative electrical permittivity. The harmonic signals injected in IP1 and IP2 are harmonic sinus of amplitude $10 \mathrm{~A}$ and variable frequency from $1 \mathrm{~Hz}$ to $300 \mathrm{MHz}$. For the pulses we used a double exponential lightning signal model.

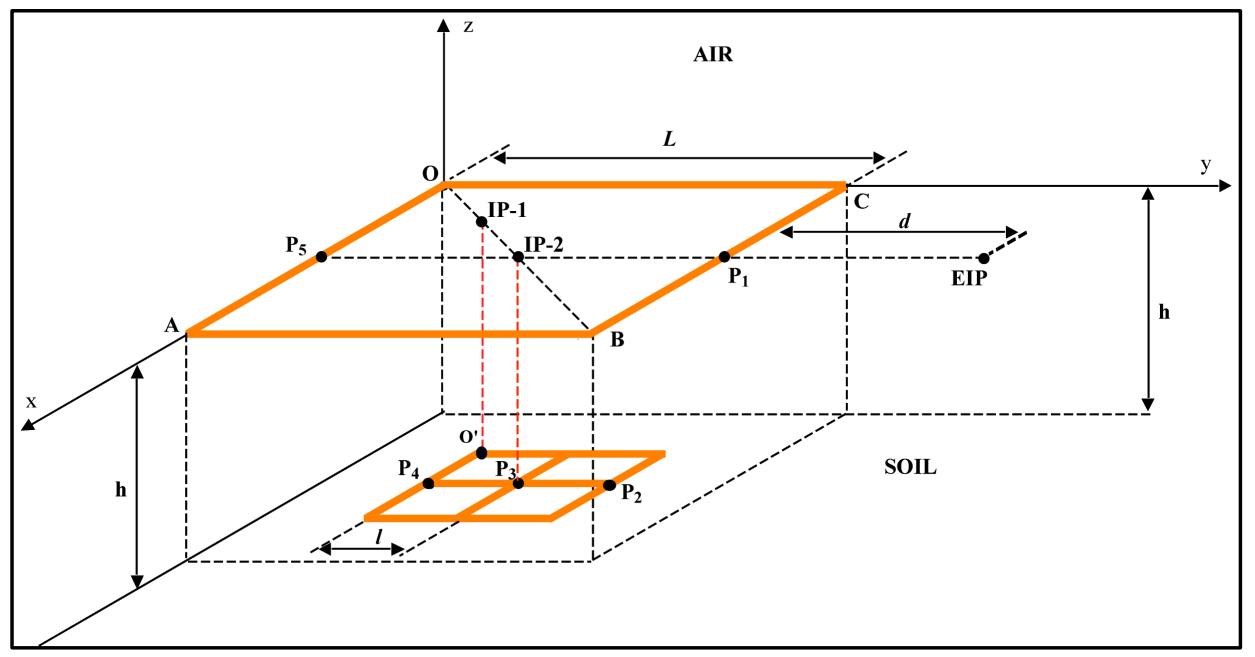

Figure 2. System of electrodes, all buried at a depth $\mathrm{h}$ in a conducting soil. The system is composed of a regular mesh half-edged 1 , and of a square conductor on the surface edged L, which simulate a metallic fence. 


$$
i(t)=I_{0}\left(e^{-\alpha t}-e^{-\beta t}\right)
$$

with the values for the constants are $\alpha=0.8 \times 10^{6} \mathrm{~s}^{-1}$ and $\beta=5.78 \times 10^{8} \mathrm{~s}^{-1}$. For the injected pulse at IP2, this immediately starts from time $t=0$ and the study covers a range of $\Delta t=3.14 \times 10^{-5}$ seconds, while for the pulse injected at EIP, it begins after a delay equivalent to $10 \%$ of the total pulse duration, and covers a range of $\Delta t=6.3 \times 10^{-6}$ seconds. The calculations are carried out with $1 / 3$ of the frequency spectrum of the pulses. Calculations have been divided into the following subsections:

\subsection{Harmonic Regime}

The active electrode is fed with a 10 A sinusoidal current and variable frequency injected through IP1 or IP2.

\subsubsection{Grounding Impedance as a Function of Frequency for the Both Feeding Points IP1 and IP2}

Defining the input impedance with respect to a feeding point, at a given value of the frequency $\omega$, as

$$
Z(\omega)=\frac{V(\omega)}{I}
$$

where $V(w)$ is the potential phasor appearing in the selected injection point, and $I$ is the current phasor applied at that point.

Figure 3 shows the results for the modulus of the grounding impedance at injection points IP1 and IP2 as a function of the frequency of the harmonic signal injected.

It should be mentioned that at low frequency, the impedance values should be independent of the injection point with significant differences at medium and high frequency. Figure 3 shows that at low frequencies, there is a small difference in impedance values approximately $10 \%$. This is explained by the segmentation of the electrodes considered here. Table 1 shows the values of the input impedance for IP1 and IP2 points at a frequency

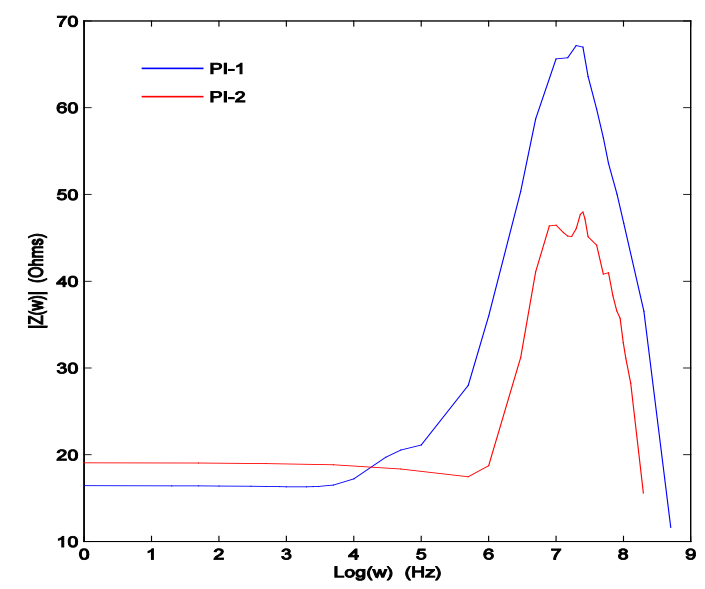

Figure 3. Absolute value of the input grounding impedance associated to injection points IP1 (blue line) and IP2 (red line), as a function of the frequency decimal logarithm.

Table 1. Input impedance (module) to the injection points IP1 and IP2 at the frequency of $50 \mathrm{~Hz}$, for several values of the partition into $\mathrm{N}$ segments of the rods.

\begin{tabular}{ccc}
\hline & IP1 & IP2 \\
\hline $\mathrm{N}$ & $|\mathrm{Z}(\omega)|$ & $|\mathrm{Z}(\omega)|$ \\
\hline 30 & 17.126 & 18.994 \\
50 & 17.889 & 18.817 \\
70 & 18.239 & 18.704 \\
90 & 18.402 & 18.524 \\
\hline
\end{tabular}


of $50 \mathrm{~Hz}$ when the segmentation is refined.

It is clear that there is a convergence towards a common value of the grounding impedance and that the differences are due to the errors arising from the numerical treatment of the problem.

\subsubsection{Harmonic Potential Values along p2 and p4 of Figure2 on the Active Electrode, When Considered IP2 as the Feeding Point}

Figure 4 shows the potential phasors along the active electrode between points $\mathrm{p} 2$ and $\mathrm{p} 4$, in Figure 2 for low and medium frequency. It is noted that at low and medium frequency the potential profiles are very similar to each other. Significant differences are found from the frequency of $1 \mathrm{MHz}$ on, at which the grounding impedance begins to grow significantly.

For high frequencies, as shown in Figure 5, the profiles are similar to each other but differ from those found

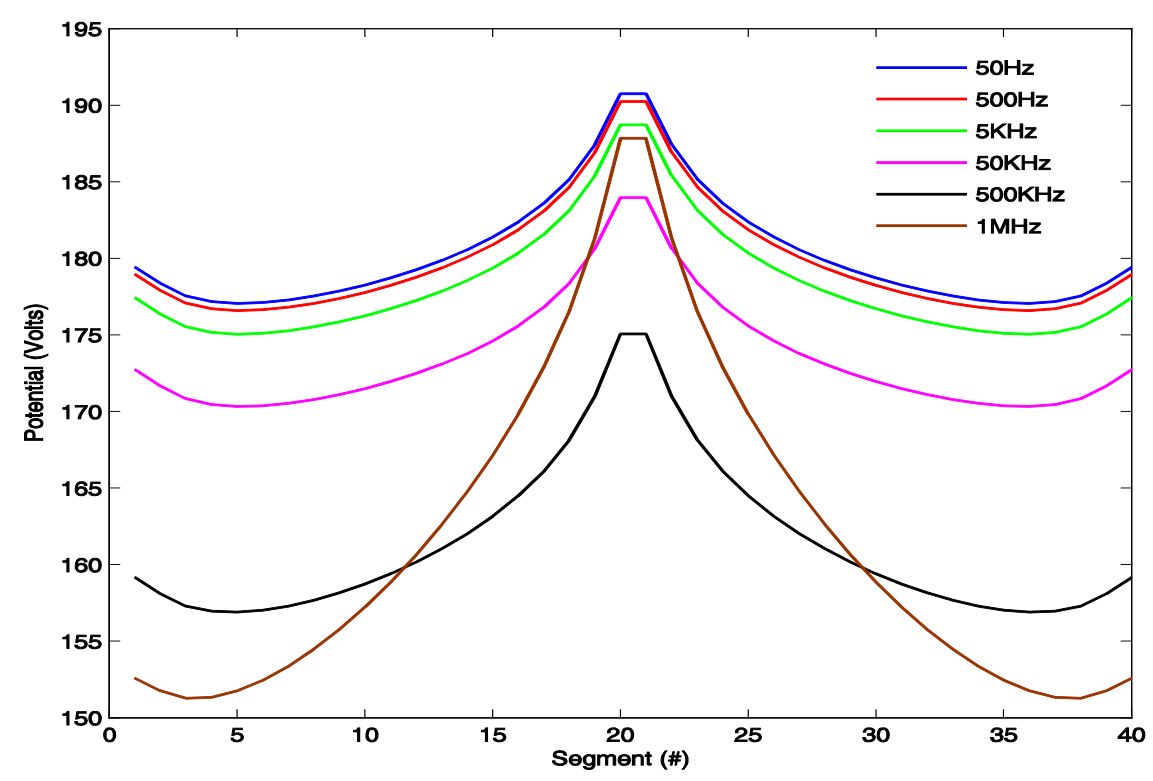

Figure 4. Potential profile along the points p2 to p4 (Figure 2) on the active electrode at the written frequencies.

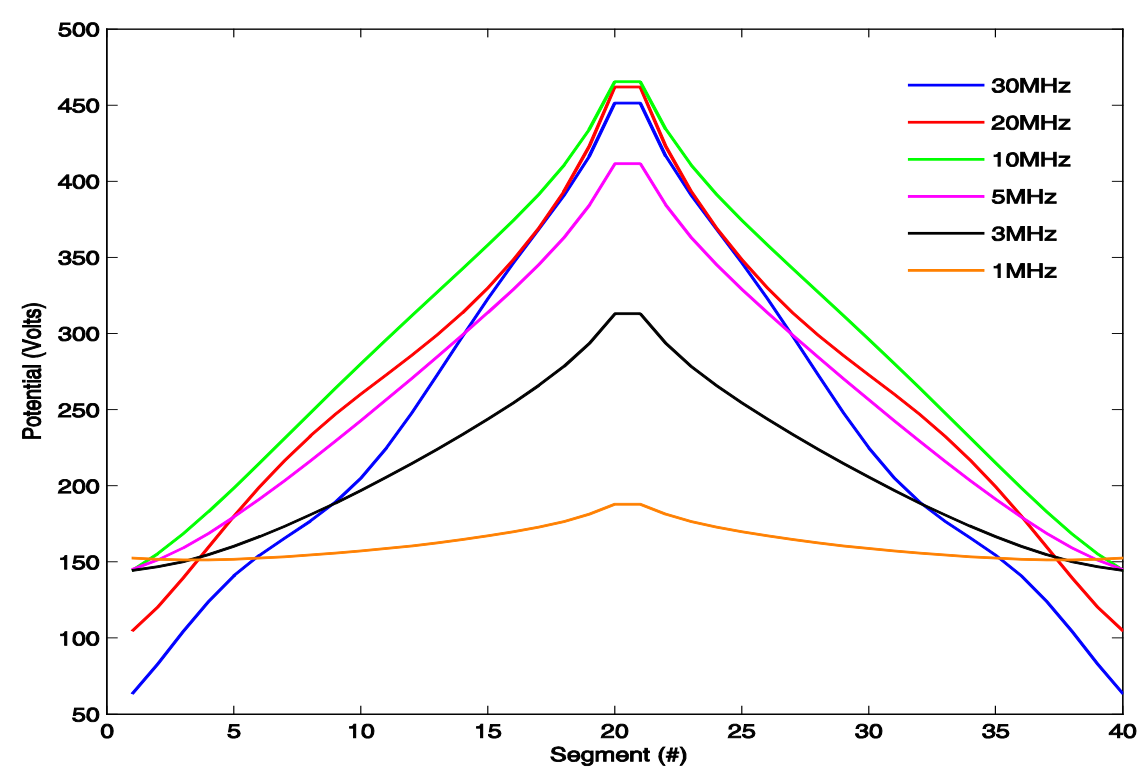

Figure 5. The same as in Figure 4 but at higher frequencies. 
at low frequencies. It is observed, that the voltage increases in the range between 1 and $10 \mathrm{MHz}$ and has a certain saturation with oscillations in the vicinity of 450 volts, which corresponds with ripples observed in Figure 3 in the mentioned frequency range.

\subsubsection{Peak Values of the Harmonic Potential in the Passive Electrode Contour OABC for IP2 as the Feeding Point}

With respect to the passive electrode, for low and medium frequency, Figure 6 shows the potential profile along the electrode contour OABC. It is noteworthy that for low and medium frequency the induced potential in the passive electrode accounts for is between $31 \%$ and $37 \%$ of the potential recorded at the feeding point.

For high frequency, Figure 7 shows the induced potential in the passive electrode. As can be seen, it is substantially lower than those recorded at low and medium frequency. In this case induced potentials account for $4 \%$ of the potential in the feeding point.

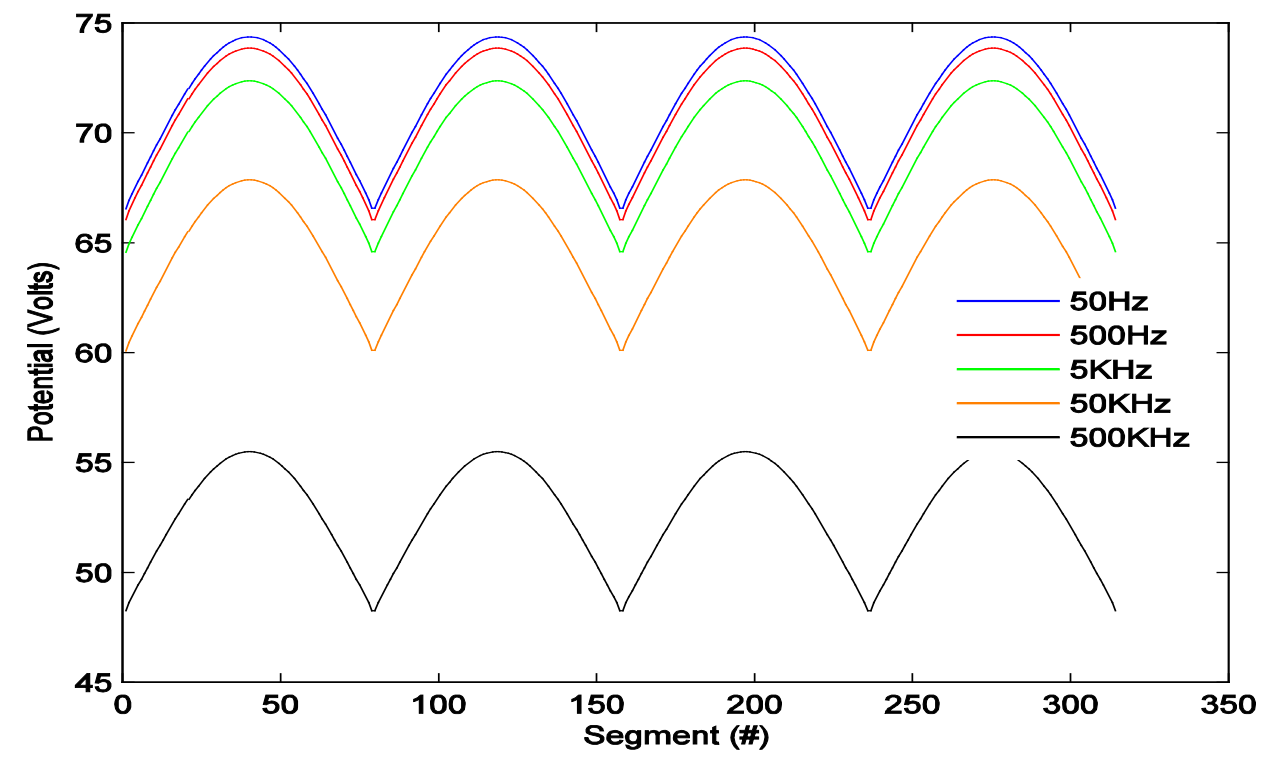

Figure 6. Potential profile along the contour $\mathrm{OABC}$ on the passive electrode at the frequencies shown.

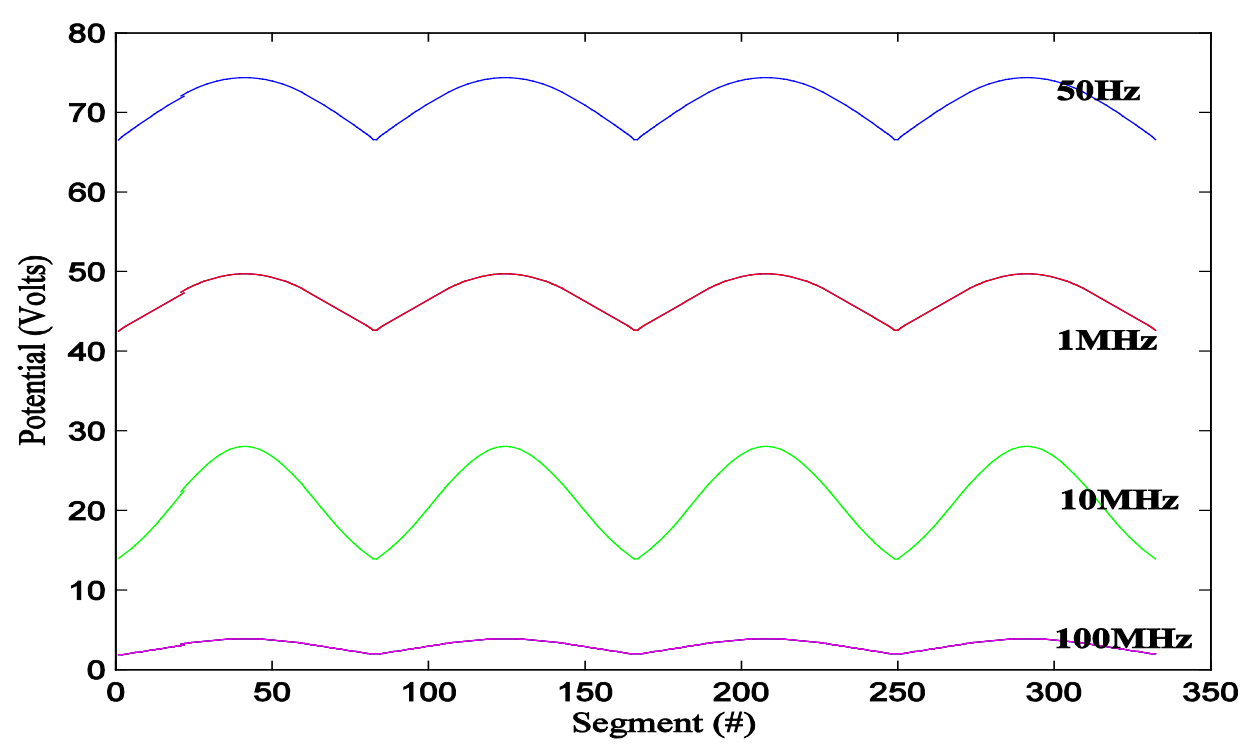

Figure 7. The same as in Figure 6, but at higher frequencies. 


\subsection{Impulse Response}

We will study two completely different situations. On one hand a peaked and short in time signal starting immediately at $t=0$ will be injected at the feeding point IP2 on the active electrode. This signal will be called lightning type 1 . On the other hand, another peaked and short in time signal starting after a delay of $\delta t=0.6 \times 10^{-6} \mathrm{~s}$, will be injected at the feeding point EIP, outside the system of conductors. This signal will be called lightning type 2 . The potential appearing at specific points on both electrodes of the system will be calculated. These potentials will be graphed as a function of time.

\subsubsection{Instantaneous Potential at Points $\mathrm{p} 3$ and $\mathrm{p} 4$ of the Active Electrode and at Point $\mathrm{p} 5$ on the Passive Electrode}

When a lightning type 1 signal starting immediately at $t=0$ is injected at IP2, an instantaneous potential appears at the points p3, p4 and p5, which is shown in Figure 8. The solid red line shows the potential at the injection point IP2 on the active electrode, whereas that the blue one shows the potential at the point $\mathrm{p} 4$ on the same electrode. They are seen to be very similar. The green solid line, however, shows the potential at the point $\mathrm{p} 5$ on the passive electrode.

It can be seen that the peak of the induced potential on the passive electrode represents approximately $37 \%$ of the peak of the potential on the active electrode which is excited by the signal. The ripples that appear in the figures are due to the finite number of Fourier components of the driving signal with which we have worked.

\subsubsection{Instantaneous Potential at Points 1 to 55 of Both Electrodes, When a Lightning Type 2}

Signal Is Injected Far from the System of Conductors at EIP (See Figure 2)

Figure 9 shows the instantaneous potential appearing at the points $\mathrm{p} 1$ to $\mathrm{p} 5$ on the electrodes of the system,

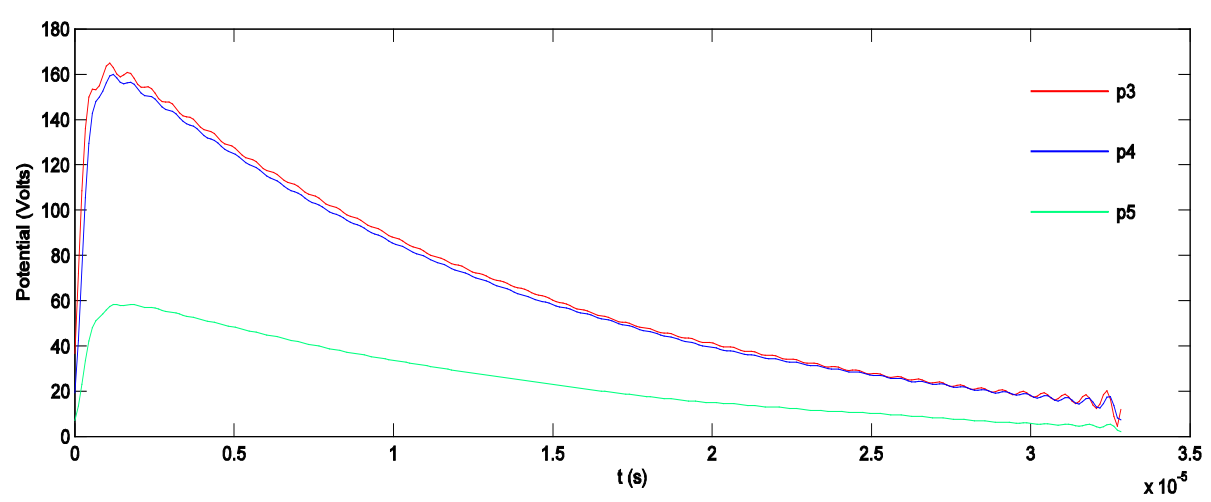

Figure 8. Instantaneous potential at points $\mathrm{p} 3$ and $\mathrm{p} 4$ on the active electrode and at point $\mathrm{p} 5$ on the passive electrode when a lightning type 1 signal is injected at IP2.

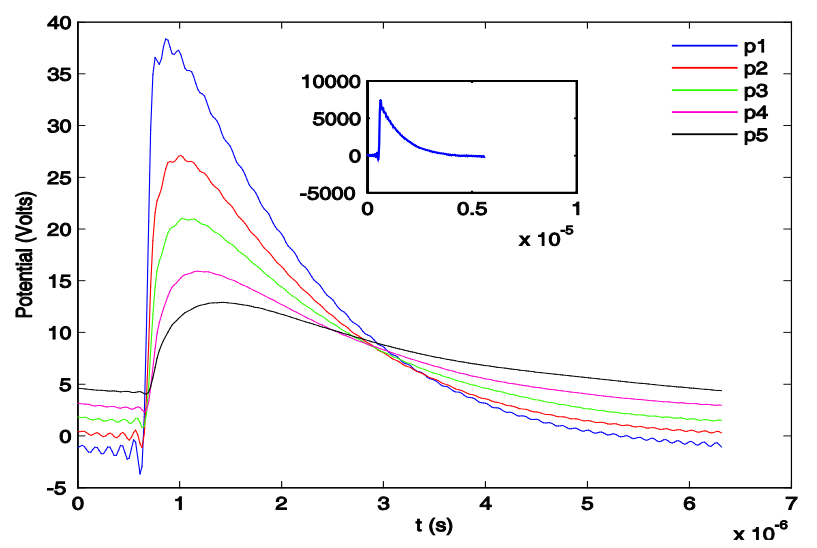

Figure 9. Instantaneous potential at points p1 to p5 on the system (Figure 2) when a lightning type 2 signal is injected at EIP at $D=2 \mathrm{~m}$. 
when the distance between the EIP point and point $\mathrm{p} 1$ on the passive electrode is $2 \mathrm{~m}$. In the subfigure, the instantaneous ground potential generated by the applied signal at the injection point EIP is represented.

When the distance $D$ (Figure 2) is increased, the induced potentials in points 1 to p5, decrease very nearly with the inverse of the distance $D$. Figure 10 shows that behavior for four values of the distance $D$.

Taking the potential peak at $\mathrm{p} 1$ as a function of the distance $D$ to point EIP, a behavior is found which, as expected, fits well with the power law.

$$
f(D)=a \cdot D^{b}+c
$$

The coefficients with $95 \%$ confidence bounds have the values $a=84.84, b=-1.029, c=-2.969$. In Figure 11, the numerical values of the potential at $\mathrm{p} 1$ as a function of $D$ and the fitted curve to the power law above mentioned are shown. By using this graph, we can estimate the fraction of the potential created at the point EIP that represents the induced potential in the passive electrode as a function of the distance $D$ between the electrode and the point EIP.

\subsubsection{Ground Potential Profile along the Diagonal OB (Figure 2), When a Lightning Type 1 Signal Is Injected at IP2}

In Figure 12, the ground potential profile along the diagonal $\mathrm{OB}$ for several time values is represented. The sequence of graphs cover the period of time, in which the potential increases from the initial value and reaches its maximum value, decreasing thereafter. The two symmetrical peaks in the figure correspond to the passive elec-
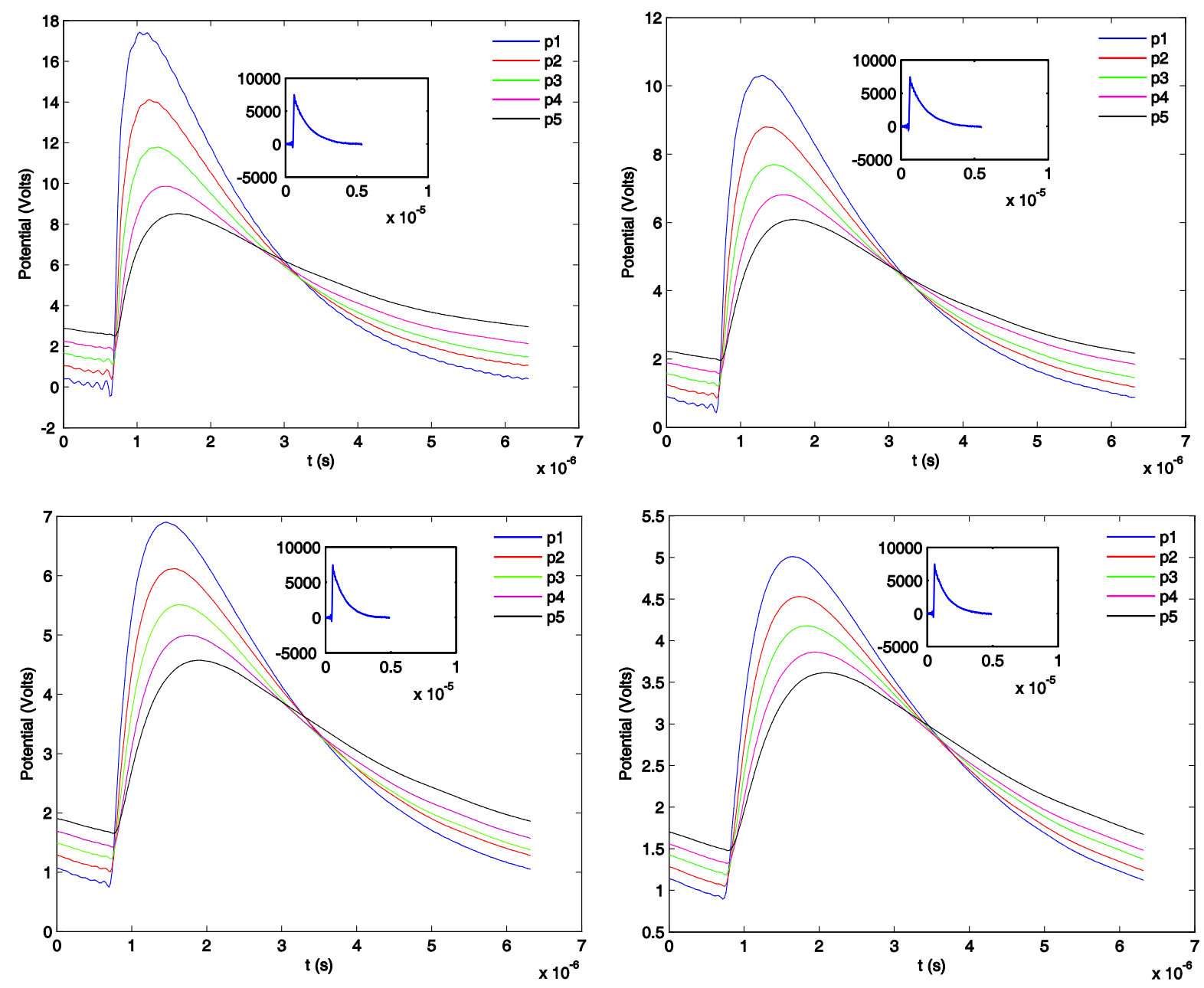

Figure 10. Instantaneous potential at points $\mathrm{p} 1$ to $\mathrm{p} 5$ (Figure 2) for $D$ ranging from 4 to $10 \mathrm{~m}$ from top to bottom and from the left to the right of the panel. 


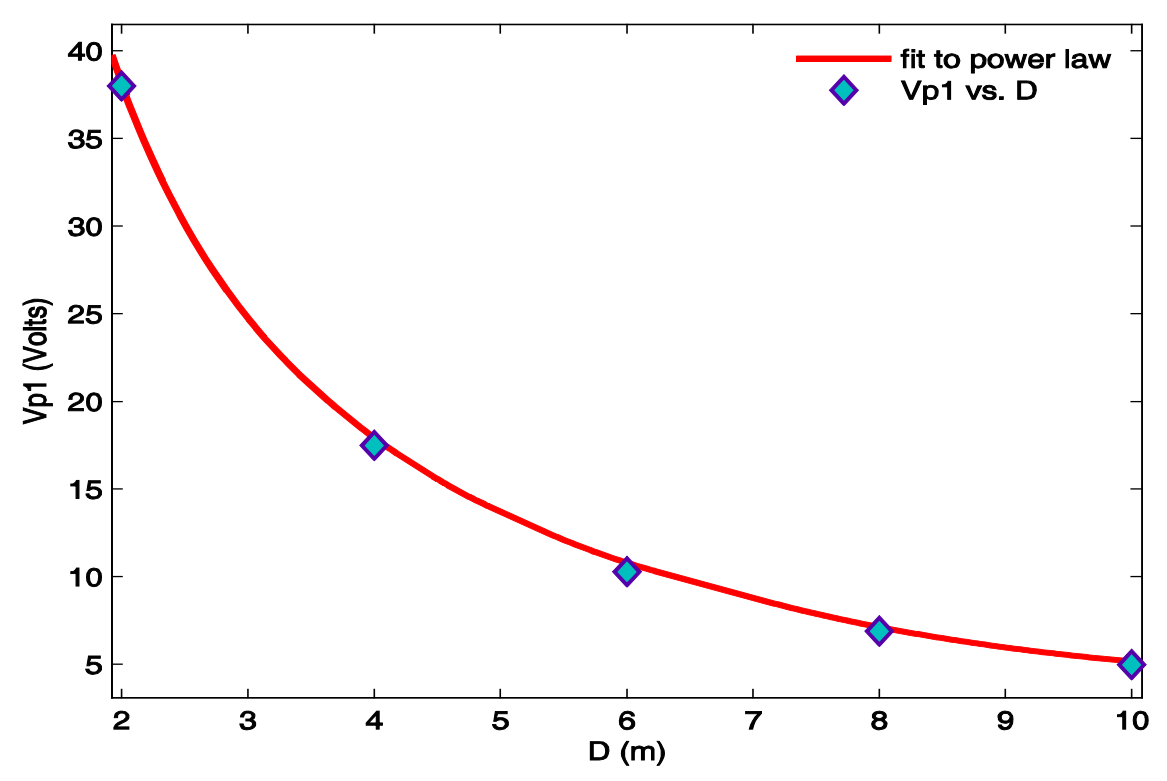

Figure 11. Potential at point $\mathrm{p} 1$ as a function of the distance $D$ (Figure 2). The fitting to a power law, whose coefficients are in the text, is shown as a continuous red line superimposed.

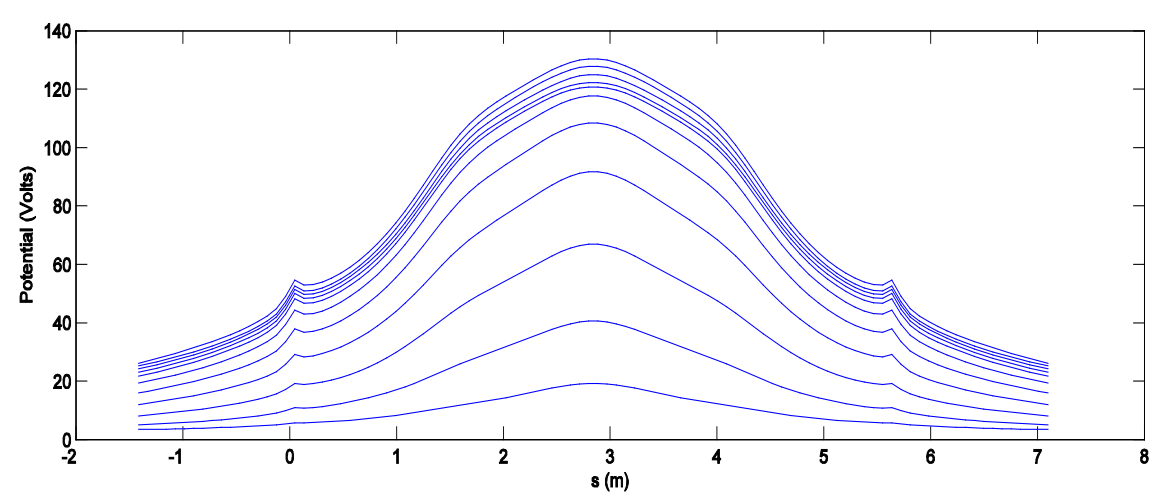

Figure 12. Ground potential profile along the diagonal OB of Figure 2. A lightning type 1 signal strikes at IP2 and a time sequence is shown from $t=0$ (line at the bottom) to the time when the ground potential reaches its maximum value.

trode potential at the points $\mathrm{O}$ and $\mathrm{B}$ because it is located on the ground.

\subsubsection{Ground Potential Profile along the Diagonal OB (Figure 2), When a Lightning Type 2 Signal Is Injected at EIP}

In Figure 13, the ground potential profile along the diagonal OB for several time values is also represented. As before, the sequence of graphs covers the period of time, in which the potential increases from the initial value and reaches its maximum value decreasing thereafter.

\section{Conclusions}

With the help of a numerical code written entirely by the authors and intended to solve the Electric Field Integral Equation (EFIE) for a thin wire structure, in this paper, we have studied the response of a system of independent electrodes to various types of driving signals. These signals are of two kinds, namely current harmonic signals injected directly on one of the electrodes, the active electrode, and lightning type signals injected at a point on the ground surface far away from the system, although they have also been directly applied to the active electrode. 


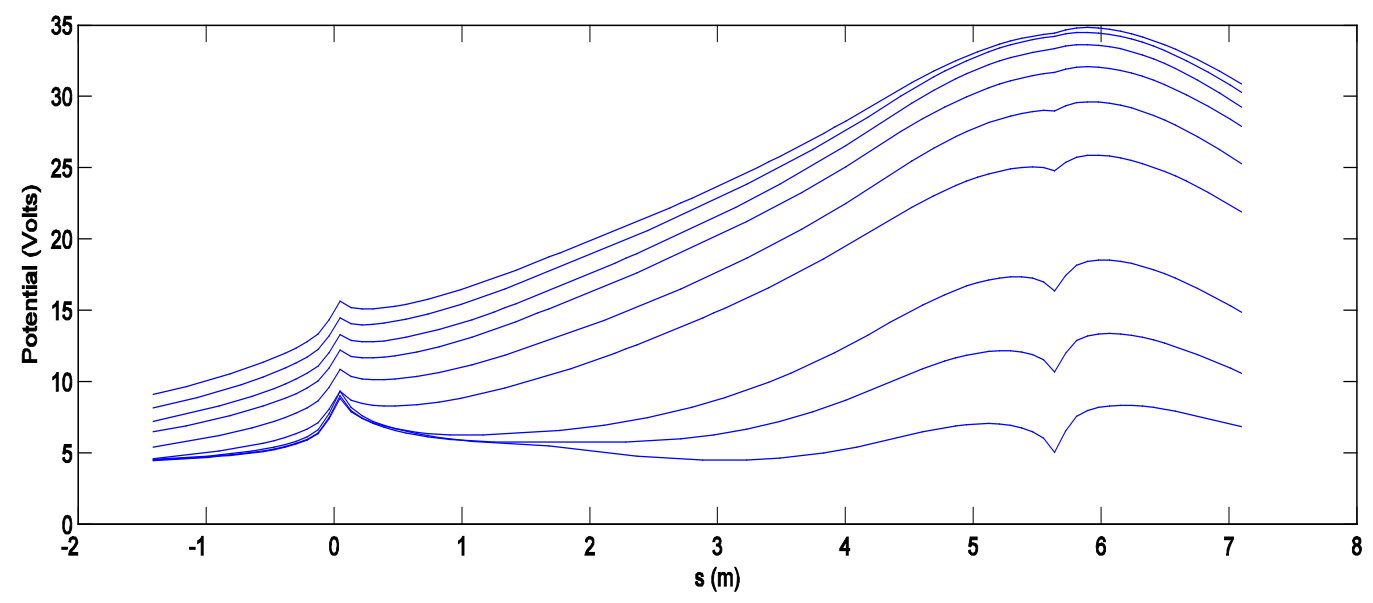

Figure 13. Ground potential profile along the diagonal OB of Figure 2. A lightning type 2 signal strikes at EIP with $D=2 \mathrm{~m}$ far from $\mathrm{p} 1$. As in Figure 12, a time sequence is shown from $\mathrm{t}=0$ (line at the bottom) to the time when the ground potential reaches its maximum value.

The response has been measured by the scalar potential generated in the electrodes of both the system and the ground surface above the buried electrodes. In particular, it has been of great interest to determine the induced potential on the electrodes not directly excited in all cases studied.

It can be stated that, regardless of the size of the electrodes of the system and their relative positions, induced potentials appearing in the electrodes may represent a significant fraction of the potential generated by the drive signal, which can pose risks for equipment and staff.

It was also concluded from the preceding analysis, that the low frequency excitations induce a greater fraction of the driving potential in the passive electrode; however, at higher frequencies the vicinity of the injection point experiences a large potential rise while it decays significantly in the passive electrode.

\section{Acknowledgements}

The authors would like to thank the Departments of Electrical Engineering, Applied Mathematics and Applied Physics of the Escuela Técnica Superior de Ingeniería y Diseño Industrial (ETSIDI) at Polytechnic University of Madrid (UPM) for their support to the undertaking of the research summarized here. Furthermore, the authors appreciate the useful suggestions and selfless assistance of Prof. P. Navarro. Finally, the authors would like to thank Ms. Gabriela Andres for her contribution to the linguistic correction of the original paper.

\section{References}

[1] Grcev, L. and Dawalibi, F. (1990) An Electromagnetic Model for Transients in Grounding Systems. IEEE Transactions on Power Delivery, 5, 1773-1781. http://dx.doi.org/10.1109/61.103673

[2] Grcev, L. (1996) Computer Analysis of Transient Voltages in Large Grounding Systems. IEEE Transactions on Power Delivery, 11, 815-823. http://dx.doi.org/10.1109/61.489339

[3] Grcev, L. (1992) Computation of Transient Voltages Near Complex Grounding Systems Caused by Lightning Currents. Symposium Record of the IEEE 1992 International Symposium on Electromagnetic Compatibility, 92CH3169-0, 393400.

[4] Vuckovic, D.D. et al. (2012) Study on a Standard for Grounding Systems Realization. Serbian Journal of Electrical Engineering, 9, 393-401. http://dx.doi.org/10.2298/SJEE1203393V

[5] Colominas, I., et al. (2012) Advanced Computer Methods for Grounding Analysis. Proceedings of the World Congress on Engineering and Computer Science 2012 (WCECS 2012), Vol. II, San Francisco, 24-26 October 2012.

[6] Cristina, S. and Orlandi, A. (1992) Calculation of the Induced Effects Due to a Lightning Stroke. Proc. Inst. Elect. Eng. $B$, 139, 374-380.

[7] Charlton, T., Davies, M. and Baudin, D. (2007) Tranfer Potentials from MV to LV Installations during an Earth Fault. CIRED, 19th International Conference on Electricity Distribution, Vienna, 21-24 May 2007, Paper No. 0805.

[8] Poljak, D., et al. (2011) Electromagnetic Field Coupling to Multiple Buried Thin Wires. Proceedings of the 10th Sym- 
posium on Electromagnetic Compatibility, (EMC, Europe 2011), York, 26-30 September 2011, 272-277.

[9] Ala, G. and Di Silvestre, M.L. (2002) A Simulation Model for Electromagnetic Transients in Lightning Protection Systems. IEEE Transactions on Electromagnetic Compatibility, 44, 539-554. http://dx.doi.org/10.1109/TEMC.2002.804773

[10] Harrington, R.F. (1993) Field Computation by Moment Methods. IEEE Press, New York. http://dx.doi.org/10.1109/9780470544631

[11] Gibson, W.C. (2008) The Method of Moments in Electromagnetics. Chapman \& Hall/CRC, Boca Raton.

[12] Wang, J.H. (1991) Generalized Moment Methods in Electromagnetics. Wiley Interscience, New York.

[13] Berberovic, S., Haznadar, Z. and Stih, Z. (2003) Method of Moments in Analysis of Grounding Systems. Engineering Analysis with Boundary Elements, 27, 351-360. http://dx.doi.org/10.1016/S0955-7997(02)00123-6

[14] Ala, G., Buccheri, P., Francomano, E. and Tortorici, A. (1994) An Advanced Algorithm for Transient Analysis of Grounding Systems by Moments Method. 2nd International Conference on Computation in Electromagnetics, London, 12-14 April 1994, 363-366.

[15] Liu, Y., Theethayi, N. and Thottappillil, R. (2005) An Engineering Model for Transient Analysis of Grounding System under Lightning Strikes: Nonuniform Transmission-Line Approach. IEEE Transactions on Power Delivery, 20, 722730. http://dx.doi.org/10.1109/TPWRD.2004.843437

[16] Geri, A. (1999) Behavior of Grounding Systems Excited by High Impulse Currents: The Model and Its Validation. IEEE Transactions on Power Delivery, 14, 1008-1017. http://dx.doi.org/10.1109/61.772347 